\title{
TITLE:
}

\section{Fine-Needle Aspirates of Thyroid Microcarcinoma}

Kristen L. Partyka, M.D. ${ }^{1}$ and Howard H. Wu, M.D. ${ }^{1}$

${ }^{1}$ Department of Pathology and Laboratory Medicine

Indiana University School of Medicine

350 W. 11th Street

Indianapolis, IN 46202

United States of America

Email:partykak@iupui.edu and hhwu@iupui.edu

RUNNING TITLE: Thyroid microcarcinoma FNA

\section{Corresponding author:}

Kristen L. Partyka, MD

Indiana University School of Medicine

IU Health Pathology Laboratory

350 W. 11th Street, IUHPL \#4086

Indianapolis, IN 46202

Telephone: $\quad 317-491-6154$

Facsimile: $\quad 317-491-6419$

Email:partykak@iupui.edu

Disclosures: There are no prior publications or submissions with any overlapping information, including studies and patients. This manuscript has not been and will not be submitted to any other journal while it is under consideration. Each author listed on the manuscript has seen and approved the submission of this version of the manuscript and takes full responsibility for the manuscript. The authors have no financial or conflicts of interest to disclose. This research received no specific grant from any funding agency in the public, commercial, or not-for-profit sectors.

Precis: In our experience, indeterminate diagnoses (FLUS+FN+SM) accounted for the majority $(60 \%)$ of preoperative FNAs for incidental microcarcinoma (size $\leq 0.5 \mathrm{~cm}$ ) when compared to $27 \%$ for those of non-incidental microcarcinoma (size $>0.5 \mathrm{~cm}$ and $\leq 1 \mathrm{~cm})(p<0.05$, chi-square test).

KEYWORDS: thyroid; FNA; cytology; the Bethesda System; microcarcinoma

This is the author's manuscript of the article published in final edited form as:

Partyka, K. L., \& Wu, H. H. (2017). Fine-Needle Aspirates of Thyroid Microcarcinoma. Journal of the American Society of Cytopathology. https://doi.org/10.1016/j.jasc.2017.06.006 


\section{ABSTRACT: \\ INTRODUCTION:}

Widespread use of ultrasound allows for detection of smaller thyroid nodules and preoperative evaluation with fine-needle aspiration (FNA). Both incidental and non-incidental microcarcinoma can be found, leading to uncertainty with clinical management.

\section{MATERIALS AND METHODS:}

A retrospective analysis of thyroid FNAs performed at our institution was conducted for the 5year period from 2010 to 2014. Aspirates were categorized using the Bethesda System for Reporting Thyroid Cytopathology. Cytologic diagnoses were then correlated with final histopathology. Among samples with malignancy on surgical resection, nodules were stratified by size.

\section{RESULTS:}

A total of 2531 thyroid FNAs were identified. 587 samples had histologic correlation, and 259 malignancies were reported. They were separated into nodules $>1 \mathrm{~cm}(\mathrm{n}=144,56 \%)$ and $\leq 1 \mathrm{~cm}$ ( $\mathrm{n}=115,44 \%$ ). Microcarcinoma was further subdivided into incidental ( $\operatorname{size} \leq 0.5 \mathrm{~cm}, \mathrm{n}=55,48 \%$ ) and non-incidental (size $>0.5 \mathrm{~cm}$ and $\leq 1 \mathrm{~cm}, \mathrm{n}=60,52 \%$ ). The preoperative cytologic diagnoses for incidental microcarcinoma were: benign $(B, n=11,20 \%)$, follicular lesion of undetermined significance (FLUS, n=15, 27\%), follicular neoplasm (FN, n=11, 20\%), suspicious for malignancy (SM, $n=7,13 \%)$, malignant $(M, n=8,15 \%)$, and non-diagnostic $(N D, n=3,5 \%)$. The FNA categories for non-incidental microcarcinoma were: B ( $n=13,22 \%)$, FLUS $(n=3,5 \%)$, FN $(\mathrm{n}=3,5 \%), \operatorname{SM}(\mathrm{n}=10,17 \%), M(\mathrm{n}=29,48 \%)$ and $\mathrm{ND}(\mathrm{n}=2,3 \%)$.

\section{CONCLUSIONS:}

Incidental microcarcinoma is not an uncommon entity, making up 21\% (55/259) of malignant nodules on thyroidectomy. Indeterminate diagnoses (FLUS+FN+SM) accounted for the majority 
(60\%) of preoperative FNAs for incidental microcarcinoma when compared to $27 \%$ for those of non-incidental microcarcinoma $(p<0.05$, chi-square test).

KEYWORDS: thyroid; FNA; cytology; the Bethesda System; microcarcinoma 


\section{MANUSCRIPT TEXT:}

\section{INTRODUCTION:}

Thyroid cancer is the most common malignancy of the endocrine system, and the incidence has dramatically increased in recent years. The majority of cases consist of papillary thyroid carcinoma (PTC), making up more than $80 \%$ of histologic diagnoses. ${ }^{1-3}$ A paramount study with the Surveillance, Epidemiology, and End Results (SEER) data exhibited a nearly threefold increase in PTC from 1973 to 2009. ${ }^{1,4}$ The bulk of this increase was shown to be associated with an upsurge in cancers of smaller size. Microcarcinoma, defined as measuring $\leq 1 \mathrm{~cm}$ by the World Health Organization, comprised $49 \%$ of the increase in incidence. ${ }^{1,5}$

It is unclear whether the rising incidence is a true epidemic of disease or reflects the enhanced detection of thyroid nodules with advanced imaging techniques. ${ }^{1,6}$ Many nodules are discovered during the use of head and neck imaging for unrelated symptoms. Widespread use of ultrasound, computed tomography, magnetic resonance imaging, and positron emission tomography allows for detection of smaller thyroid nodules. Ultrasound imaging can detect nodules as small as 0.2 $\mathrm{cm}$ in dimension, but the clinical significance of these findings is highly debated. ${ }^{1,7}$

Microcarcinoma is generally thought to have an indolent nature, presenting as an ancillary finding at the time of histologic examination. Foci of papillary thyroid microcarcinoma have been reported in up to $22 \%$ of surgical thyroid specimens and up to $36 \%$ of autopsy series. ${ }^{6,8-11}$ However, its biologic behavior can be variable with associated morbidity and mortality. Some patients have local lymph node metastases or distant metastases at the time of diagnosis or during years of follow-up., ${ }^{72-13}$ Other authors have even reported cancer-related deaths. ${ }^{14}$ 
A pioneer study in 1987 described a higher rate of lymph node metastasis in patients with thyroid microcarcinoma $>0.5 \mathrm{~cm}$ when compared to microcarcinoma $\leq 0.5 \mathrm{~cm} .{ }^{15}$ Since that publication, many research groups have examined the significance of various clinicopathologic risk factors with an established size cutoff of $0.5 \mathrm{~cm} .{ }^{16-19}$ One study corroborated the finding of an increased incidence of lymph node metastasis in patients with microcarcinoma $>0.5 \mathrm{~cm}$, supporting the importance of this size distinction. ${ }^{16}$ A second publication went further to report the cutoff of $>0.5 \mathrm{~cm}$ to be an independent predictor of subclinical central lymph node metastasis. ${ }^{19}$ Another study found a significant difference in extrathyroidal extension in patients with microcarcinoma $>0.5 \mathrm{~cm} .{ }^{17}$ There is potential value in using $0.5 \mathrm{~cm}$ as a size parameter when analyzing microcarcinoma.

Standard of care for thyroid cancer and lymph node involvement is total thyroidectomy with central lymph node dissection. ${ }^{19}$ However, the rise in both incidental and non-incidental microcarcinoma has generated controversy surrounding optimal management. There is little epidemiologic data on the broad spectrum of disease characteristics and long-term prognosis of microcarcinoma. ${ }^{20}$ Recommendations for clinical management range from observation alone to total thyroidectomy with lymph node dissection and radioactive iodine..$^{2,8,12,20-22}$

Ultrasound-guided fine-needle aspiration (FNA) is a fundamental component to the preoperative evaluation of thyroid nodules. It is a safe and minimally invasive modality, separating nodules into well-defined diagnostic tiers to steer appropriate patient care. ${ }^{9}$ Nevertheless, there is little known about the relationship between microcarcinoma and cytologic categories on FNA. 
Microcarcinoma has a clear impact on the increasing incidence of thyroid cancer. We sought to investigate the correlation between microcarcinoma and preoperative cytology to facilitate communication with clinicians.

\section{MATERIALS AND METHODS:}

This study was approved by the Institutional Review Board of Indiana University. A retrospective analysis of thyroid FNAs performed at our institution was conducted for the 5-year period from 2010 to 2014. All aspirates were categorized into six groups using the Bethesda System for Reporting Thyroid Cytopathology (TBSRTC). ${ }^{23}$

A computer search of the anatomic pathology archives and the electronic medical record was performed to document the histologic results of patients who underwent surgery. The cytologic diagnoses were then correlated with the final histopathology. In cases with multiple nodules, preoperative FNAs were matched with corresponding histology based on the location in the thyroid gland when that information was available. Identification of biopsy related changes in direct association with nodules was beyond the scope of this retrospective study.

Among the samples with malignancy on surgical resection, nodules were stratified by size. According to the criteria outlined by the World Health Organization, thyroid microcarcinoma was designated as $1 \mathrm{~cm}$ or less in greatest dimension. ${ }^{5}$ To further classify microcarcinoma, there are different definitions for incidental and non-incidental microcarcinoma in the literature. For our statistical analysis, we used size as the deciding factor. Previous studies highlight tumor size as an important clinicopathologic factor, but various size cutoffs have been applied. There are 
reports of higher rates of lymph node metastases and extrathyroidal extension in cases $>0.5$

cm. ${ }^{15-19}$ It provides support to our designation of $0.5 \mathrm{~cm}$ as a reasonable size cutoff. In our study, malignant nodules $\leq 0.5 \mathrm{~cm}$ were classified as incidental microcarcinoma. In contrast, nodules $>0.5 \mathrm{~cm}$ and $\leq 1 \mathrm{~cm}$ were delineated as non-incidental microcarcinoma.

In order to address the recent revision in nomenclature, the cases previously diagnosed as encapsulated follicular variant of papillary thyroid carcinoma (EFVPTC) were reassessed. Two cases originated at an outside institution, and these slides were not available for review. The reported diagnoses were maintained for our analysis. All other slides were examined by a boardcertified pathologist (HHW). The prevailing diagnostic criteria for noninvasive follicular thyroid neoplasm with papillary-like nuclear features (NIFTP) were applied as necessary. ${ }^{24}$

A chi-square test using an online calculator was performed to compare the rate of indeterminate FNA diagnoses between the group of incidental microcarcinoma and non-incidental microcarcinoma. The result was found to be significant at $p<0.05$ (http://www.socscistatistics.com/tests/chisquare/Default.aspx).

\section{RESULTS:}

A total of 2531 thyroid FNA samples were identified, and 587 FNAs had surgical follow-up with histologic correlation. 259 malignant thyroid nodules were found in 220 patients ( 167 females, 53 males) with an age range of 16 to 88 years. They were associated with preoperative cytologic diagnoses as follows: benign $(\mathrm{B}, \mathrm{n}=27,10 \%)$, follicular lesion of undetermined significance 
(FLUS, $n=40,15 \%$ ), follicular neoplasm ( $F N, n=23,9 \%$ ), suspicious for malignancy (SM, n=36, 14\%), malignant (M, $\mathrm{n}=126,49 \%)$, and non-diagnostic (ND, $n=7,3 \%$ ) (Table 1).

Malignancies were separated by size into nodules $>1 \mathrm{~cm}(\mathrm{n}=144,56 \%)$ and microcarcinomas $\leq 1$ $\mathrm{cm}(\mathrm{n}=115,44 \%)$. With the nodules $>1 \mathrm{~cm}$, final histopathology showed evidence of sizes ranging from 1.1 to $13 \mathrm{~cm}$ in greatest dimension (Table 1). Among cases of thyroid microcarcinoma, the nodules were 0.1 to $1.0 \mathrm{~cm}$ in size.

With the initial separation by size, malignant nodules $>1 \mathrm{~cm}$ correlated with the following preoperative cytologic diagnoses: B $(n=3,2 \%)$, FLUS $(n=22,15 \%), F N(n=9,6 \%), S M(n=19$, $13 \%), M(n=89,62 \%)$, and ND ( $\mathrm{n}=2,1 \%)$ (Table 1).

Microcarcinoma was further subdivided into incidental microcarcinoma (size $\leq 0.5 \mathrm{~cm}, \mathrm{n}=55$, $48 \%$ ) and non-incidental microcarcinoma (size $>0.5 \mathrm{~cm}$ and $\leq 1 \mathrm{~cm}, \mathrm{n}=60,52 \%$ ) (Table 1 ). The corresponding preoperative cytologic diagnoses for incidental microcarcinoma were: $\mathrm{B}(\mathrm{n}=11$, $20 \%)$, FLUS $(n=15,27 \%), F N(n=11,20 \%), \operatorname{SM}(n=7,13 \%), M(n=8,15 \%)$, and ND $(n=3,5 \%)$ (Table 1). The cytologic diagnoses for non-incidental microcarcinoma were: $B(n=13,22 \%)$, FLUS ( $n=3,5 \%), F N(n=3,5 \%), S M(n=10,17 \%), M(n=29,48 \%)$ and ND (n=2, 3\%) (Table 1). According to the new paradigm shift, reclassification of cases revealed 10 nodules $>1 \mathrm{~cm}$ $(10 / 144,7 \%)$ met the diagnostic criteria for NIFTP. Malignancies $>1 \mathrm{~cm}$ subsequently included classic papillary thyroid carcinoma (PTC, 91/134, 68\%), invasive follicular variant of papillary thyroid carcinoma (FVPTC, 20/134, 15\%), follicular carcinoma (11/134, 8\%), anaplastic 
carcinoma $(8 / 134,6 \%)$, and metastatic renal cell carcinoma $(4 / 134,3 \%)$. These nodules correlated with the following cytologic diagnoses: B $(n=1,1 \%)$, FLUS $(n=17,13 \%), F N(n=8$, $6 \%), \operatorname{SM}(\mathrm{n}=18,13 \%), \mathrm{M}(\mathrm{n}=89,66 \%)$, and ND $(\mathrm{n}=1,1 \%)$ (Table 2$).$

With the non-incidental micronodules $(>0.5 \mathrm{~cm}$ and $\leq 1 \mathrm{~cm}), 4$ cases could be categorized as NIFTP $(4 / 60,7 \%)$ (Table 3). After exclusion of NIFTP, non-incidental microcarcinoma comprised PTC (38/56, 68\%), FC (2/56, 4\%), FVPTC (14/56, 25\%), tall cell variant of papillary thyroid carcinoma (TCV , 1/56, 2\%), and medullary carcinoma (MTC, 1/56, 2\%) (Table 3). They corresponded with the following FNA diagnoses: B ( $n=12,21 \%)$, FLUS ( $n=2,4 \%), F N(3$, $\mathrm{n}=5 \%), \mathrm{SM}(\mathrm{n}=9,16 \%), \mathrm{M}(\mathrm{n}=29,52 \%)$, and ND $(\mathrm{n}=1,2 \%)$ (Table 2$).$

Incidental microcarcinoma $\leq 0.5 \mathrm{~cm}$ consisted of PTC $(\mathrm{n}=32,58 \%)$, FVPTC $(\mathrm{n}=20,36 \%)$, and MTC $(n=3,6 \%)$ (Table 4). In the category of FVPTC, a total of 10 cases were partially encapsulated (10/20, 50\%), and 7 had no capsule (7/20, 35\%). None of these 17 cases showed evidence of lymphovascular invasion or extrathyroidal extension; however, they are too small to be classified as NIFTP. One case displayed minimal capsular invasion. Furthermore, one case demonstrated a concurrent lymph node metastasis, and one case had lymphovascular invasion. Of note, the case with lymph node metastasis had a clinical history of classic papillary thyroid carcinoma on the contralateral side, status post hemithyroidectomy. Also, the case with lymphovascular invasion had a synchronous papillary thyroid carcinoma on the opposite side. The presence of a metastasis cannot be completely excluded in these two scenarios. Lastly, one case originated at an outside institution, and the slides were not available for review. The 
reported diagnosis was maintained for our analysis. Due to limitations in this evaluation, the overall number of NIFTP is likely higher than reported.

\section{DISCUSSION:}

Among prognostic factors related to thyroid carcinoma, the most controversial aspect is the tumor size. Most cases of microcarcinoma have an indolent clinical course, and some clinicians believe nodules $\leq 1 \mathrm{~cm}$ should not be biopsied. ${ }^{7}$ However, there is a subset of patients with aggressive disease who present with bulky lymph node involvement and/or distant metastases. $^{7,12-14}$

Fine-needle aspiration is indispensable to the evaluation of thyroid nodules, and it facilitates risk stratification of patients. Little data has been published on the relationship between microcarcinoma and the preoperative classification according to TBSRTC. We sought to evaluate the cytologic diagnoses of thyroid FNAs and correlate it with the final histopathology of microcarcinoma.

While the majority of malignant nodules had a size $>1 \mathrm{~cm}(144 / 259,56 \%)$, the presence of microcarcinoma was also evident in our population $(115 / 259,44 \%)$ (Table 1). Incidental thyroid microcarcinoma with size $\leq 0.5 \mathrm{~cm}$ is not an uncommon entity. It accounted for $21 \%(55 / 259)$ of malignant nodules that were discovered on follow-up thyroidectomy. It was similar in value to non-incidental microcarcinoma ( size $>0.5 \mathrm{~cm}$ and $\leq 1 \mathrm{~cm})(23 \%, 60 / 259)$ (Table 1$)$. 
Not surprisingly, most malignant nodules $>1 \mathrm{~cm}$ were associated with the cytologic category of malignant $(89 / 144,62 \%)$ (Table 1). Nevertheless, this did not hold true with microcarcinoma in our population. Indeterminate categories (FLUS+FN+SM) accounted for the majority $(60 \%)$ of preoperative FNA diagnoses for incidental microcarcinoma when compared to $27 \%$ for those of non-incidental microcarcinoma ( $p<0.05$, chi-square test) (Table 1$)$.

This data was compiled and analyzed before the recent publication supporting a paradigm shift to revise the nomenclature used in thyroid pathology. After gathering our original data, a second tier of analysis was performed to address the new terminology of NIFTP. ${ }^{24}$ The inclusion criteria were created based on evaluation of tumors $\geq 1 \mathrm{~cm}$, yet there was no minimum size established for future application of the diagnosis. ${ }^{25}$ To thoroughly explore the issue, we examined tumors of all sizes with the diagnosis of EFVPTC in the final pathology report.

The task of reclassifying the malignancies generated only a slight adjustment in the statistical results. Of the original 204 malignant nodules that were $>0.5 \mathrm{~cm}$, review of the cases of EFVPTC revealed only 14 cases $(7 \%)$ met the criteria to now be defined as NIFTP. ${ }^{24}$ In our population, most of these cases were $>1 \mathrm{~cm}$ in size $(10 / 14)$. The remaining cases were nonincidental micronodules with size $>0.5 \mathrm{~cm}$ and $\leq 1 \mathrm{~cm}$ (4/14) (Table 3). None of the incidental microcarcinomas necessitated a new classification (Table 4).

Several recent studies on the characteristics of thyroid microcarcinoma and aspects associated with aggressive disease found size to be an important risk factor. In one retrospective cohort, microcarcinoma $\geq 0.8 \mathrm{~cm}$ was shown to be associated with neck lymph node involvement and 
distant metastases at presentation and recurrence of disease at follow-up. ${ }^{11}$ Another study uncovered increased risk for central lymph node metastases in tumors $\geq 0.6 \mathrm{~cm} .{ }^{26}$ Lastly, longterm follow-up in Japan found patients with tumor size of 0.1-0.5 cm had an improved recurrence-free survival rate 35 years after surgery when compared to patients with tumor size of $0.6-1.0 \mathrm{~cm}^{20}$

The exact size predictive of aggressive disease in microcarcinoma appears to slightly vary with the study population. Our distinction of non-incidental microcarcinoma $(>0.5 \mathrm{~cm}$ and $\leq 1 \mathrm{~cm})$ and incidental microcarcinoma $(\leq 0.5 \mathrm{~cm})$ is in accordance with the separation points used in other analyses. ${ }^{15-20,26}$ After exclusion of NIFTP, the indeterminate categories (FLUS+FN+SM) still accounted for the majority (60\%) of preoperative FNA diagnoses for incidental microcarcinoma when compared to $25 \%$ for those of non-incidental microcarcinoma ( $p<0.05$, chi-square test) (Table 2).

These results can presumably be attributed to a sampling issue inherent to the procedure and not solely an interpretation error. The cytologic diagnoses were rendered by multiple different boardcertified pathologists and often with use of intradepartmental consensus. With our designation of $\leq 0.5 \mathrm{~cm}$ as incidental microcarcinoma, it is reasonable to surmise that many of these were indeed incidental findings and too small to be adequately sampled by fine-needle aspiration. A sample composed of atypical cells admixed with normal follicular cells would result in an indeterminate diagnosis on FNA. Among the cases with a cytologic diagnosis of either FLUS or FN (26 cases, 47\%), all of the cases showed benign nodules (adenomas, hyperplastic nodules, and lymphocytic thyroiditis) adjacent to the incidental microcarcinoma in the resection specimens. However, in 8 
cases $(15 \%)$, size ranging from $0.3 \mathrm{~cm}$ to $0.5 \mathrm{~cm}$, there were sufficient cellular changes for us to render a definite malignant diagnosis.

Earlier studies indicated that microcarcinoma was an entity mostly discovered in thyroid glands removed for benign disease or at the time of autopsy. Recent trends in cancer surveillance and advanced diagnostic techniques have contributed to a shift in size distribution toward smaller tumors. ${ }^{1,4,27}$ Due to the uncertain behavior of microcarcinoma, the use of FNA on thyroid nodules $\leq 1 \mathrm{~cm}$ has been a frequent topic of debate. ${ }^{10}$ The 2015 American Thyroid Association guidelines discourage using FNA to evaluate subcentimeter thyroid nodules in patients without evidence of extrathyroidal extension or a sonographically suspicious lymph node. ${ }^{28}$ However, current recommendations justify the use of FNA to assess nodules $\leq 1 \mathrm{~cm}$ with worrisome features such as hypoechogenicity, irregular margins, and microcalcifications on ultrasound imaging. ${ }^{9-10}$

Regardless of size, thyroid nodules with suspicious findings can be subjected to preoperative evaluation with fine-needle aspiration. Our analysis highlights the incidence of microcarcinoma and its correlation with each FNA category. Although the cytology smears may not be definitive, this should not deter clinicians from using fine-needle aspiration to assess smaller nodules. Imaging characteristics, patient demographics, and clinical risk factors all play a role in management. Knowing that microcarcinoma can contribute to an indeterminate diagnosis creates an indispensable platform for clinicians to discuss treatment options with patients. When an indeterminate diagnosis is rendered on FNA, microcarcinoma is a legitimate possibility. Active surveillance is a viable approach for patients with papillary microcarcinoma, but we propose that surgical resection such as lobectomy may be a better option for patients who are good surgical 
candidates. Understanding the prominence of microcarcinoma associated with each cytologic diagnosis is instrumental to guide optimal clinical management. 


\section{REFERENCES:}

1. Davies L, Welch HG. Increasing incidence of thyroid cancer in the United States, 1973-2002. JAMA. 2006; 295: 2164-2167.

2. Karatzas T, Vasileiadis I, Kapetanakis S, Karakostas E, Chrousos G, Kouraklis G. Risk factors contributing to the difference in prognosis for papillary versus micropapillary thyroid carcinoma. Am J Surg. 2013; 206: 586-593.

3. Provenzale MA, Fiore E, Ugolini C, et al. 'Incidental' and 'non-incidental' thyroid papillary microcarcinomas are two different entities. Eur J Endocrinol. 2016; 174: 813-820.

4. Davies L, Welch HG. Current thyroid cancer trends in the United States. JAMA Otolaryngol Head Neck Surg. 2014; 140: 317-322.

5. Hedinger C, Williams ED, Sobin LH. The WHO histological classification of thyroid tumors: a commentary on the second edition. Cancer. 1989; 63: 908-911.

6. Zevallos JP, Hartman CM, Kramer JR, Sturgis EM, Chiao EY. Increased thyroid cancer incidence corresponds to increased use of thyroid ultrasound and fine-needle aspiration: a study of the Veterans Affairs health care system. Cancer. 2015; 121: 741-746.

7. Yang GCH, LiVolsi VA, Baloch ZW. Thyroid microcarcinoma: fine-needle aspiration diagnosis and histologic follow-up. Int J Surg Pathol. 2002; 10: 133-139.

8. Elliott MS, Gao K, Gupta R, Chua EL, Gargya A, Clark J. Management of incidental and non-incidental papillary thyroid microcarcinoma. J Laryngol Otol. 2013; 127: S17-S23.

9. Unal B, Sezer C. Diagnostic value of ultrasound-guided fine needle aspiration biopsy in malignant thyroid nodules: utility for micronodules. Asian Pac J Cancer Prev. 2014; 15: 8613-8616. 
10. Barbaro D, Simit U, Meucci G, Lapi P, Orsini P, Pasquini C. Thyroid papillary cancers: microcarcinoma and carcinoma, incidental cancers and non-incidental cancers - are they different diseases? Clinical Endocrinology. 2005; 63: 577-581.

11. Roti E, Rossi R, Trasforini G, et al. Clinical and histological characteristics of papillary thyroid microcarcinoma: results of a retrospective study in 243 patients. J Clin Endocrinol Metab. 2006; 91: 2171-2178.

12. Sugitani I, Fujimoto Y. Symptomatic versus asymptomatic papillary thyroid microcarcinoma: a retrospective analysis of surgical outcome and prognostic factors. Endocr J. 1999; 46: 209-216.

13. Jedrzkiewicz J, Witt BL. FNA diagnosis of a metastatic papillary thyroid carcinoma arising from a previously unknown follicular variant of papillary thyroid microcarcinoma. Diagn Cytopathol. 2014; 42: 711-715.

14. Noguchi S, Yamashita H, Murakami N, Nakayama I, Toda M, Kawamoto H. Small carcinomas of the thyroid: a long-term follow-up of 867 patients. Arch Surg. 1996; 131: $187-$ 191.

15. Kasai N, Sakamoto A. New subgrouping of small thyroid carcinomas. Cancer. 1987; 60: 1767-1770.

16. Wada N, Duh QY, Sugino K, et al. Lymph node metastasis from 259 papillary thyroid microcarcinomas: frequency, pattern of occurrence and recurrence, and optimal strategy for neck dissection. Ann Surg. 2003; 237: 399-407.

17. Chow SM, Law SCK, Chan JKC, Au SK, Yau S, Lau WH. Papillary microcarcinoma of the thyroid—prognostic significance of lymph node metastasis and multifocality. Cancer. 2003; 98: $31-40$. 
18. Vasileiadis I, Karakostas E, Charitoudis G, et al. Papillary thyroid microcarcinoma: clinicopathological characteristics and implications for treatment in 276 patients. Eur J Clin Invest. 2012; 42: 657-664.

19. Kim JY, Jung EJ, Park T, et al. Impact of tumor size on subclinical central lymph node metastasis in papillary thyroid microcarcinoma depends on age. World J Surg Oncol. 2015; 13: 88 .

20. Noguchi S, Yamashita H, Uchino S, Watanabe S. Papillary microcarcinoma. World J Surg. 2008; 32: 747-753.

21. McDougall IR, Camargo CA. Treatment of micropapillary carcinoma of the thyroid: where do we draw the line? Thyroid. 2007; 17: 1093-1096.

22. Wang SF, Zhao WH, Wang WB, Teng XD, Teng LS, Ma ZM. Clinical features and prognosis of patients with benign thyroid disease accompanied by an incidental papillary carcinoma. Asian Pacific J Cancer Prev. 2013; 14: 707-711.

23. Cibas ES, Ali SZ. The Bethesda system for reporting thyroid cytopathology. Am J Clin Pathol. 2009; 132: 658-665.

24. Nikiforov YE, Seethala RR, Tallini G, et al. Nomenclature revision for encapsulated follicular variant of papillary thyroid carcinoma: a paradigm shift to reduce overtreatment of indolent tumors. JAMA Oncol. 2016; 2: 1023-1029.

25. Thompson LDR. Update on follicular variant of papillary thyroid carcinoma with an emphasis on new terminology: noninvasive follicular thyroid neoplasm with papillary-like nuclear features. Diagn Histopathol. 2016; 22: 171-178.

26. Zhang LY, Liu ZW, Liu YW, Gao WS, Zheng CJ. Risk factors for nodal metastasis in cN0 papillary thyroid microcarcinoma. Asian Pac J Cancer Prev. 2015; 16: 3361-3363. 
27. Hughes DT, Haymart MR, Miller BS, Gauger PG, Doherty GM. The most commonly occurring papillary thyroid cancer in the United States is now a microcarcinoma in a patient older than 45 years. Thyroid. 2011; 21: 231-236.

28. Haugen BR, Alexander EK, Bible KC, et al. 2015 American Thyroid Association management guidelines for adult patients with thyroid nodules and differentiated thyroid cancer: the American Thyroid Association guidelines task force on thyroid nodules and differentiated thyroid cancer. Thyroid. 2016; 26: 1-133. 
TABLES:

Table 1. FNA Diagnosis and Malignant Nodules on Surgical Follow-Up

\begin{tabular}{|c|c|c|c|}
\hline FNA Diagnosis & $\mathbf{5 0 . 5} \mathbf{~ c m}$ & $\mathbf{> 0 . 5} \mathbf{~ c m}$ and $\mathbf{1} \mathbf{~ c m}$ & $\mathbf{> 1} \mathbf{~ c m}(1.1-13 \mathbf{c m})$ \\
\hline B $(\mathrm{n}=27,10 \%)$ & $11(20 \%)$ & $13(22 \%)$ & $3(2 \%)$ \\
\hline FLUS $(\mathrm{n}=40,15 \%)$ & $15(27 \%)$ & $3(5 \%)$ & $22(15 \%)$ \\
\hline FN $(\mathrm{n}=23,9 \%)$ & $11(20 \%)$ & $3(5 \%)$ & $9(6 \%)$ \\
\hline SM $(\mathrm{n}=36,14 \%)$ & $7(13 \%)$ & $10(17 \%)$ & $19(13 \%)$ \\
\hline $\mathbf{M}(\mathrm{n}=126,49 \%)$ & $8(15 \%)$ & $29(48 \%)$ & $89(62 \%)$ \\
\hline ND $(\mathrm{n}=7,3 \%)$ & $3(5 \%)$ & $2(3 \%)$ & $2(1 \%)$ \\
\hline Total $(\mathrm{n}=259)$ & 55 & 60 & 144 \\
\hline
\end{tabular}

$\mathrm{B}=$ Benign; FLUS=Follicular lesion of undetermined significance; FN=Follicular neoplasm; $\mathrm{SM}=$ Suspicious for malignancy; $\mathrm{M}=$ Malignant; $\mathrm{ND}=$ Non-diagnostic 
Table 2. FNA Diagnosis and Malignant Nodules after Exclusion of NIFTP

\begin{tabular}{|c|c|c|}
\hline FNA Diagnosis & $\mathbf{> 0 . 5} \mathbf{~ c m}$ and $\mathbf{~ 1 ~ c m ~}$ & $>\mathbf{1 ~ c m ~}(1.1-13 \mathbf{c m})$ \\
\hline B $(\mathrm{n}=13,7 \%)$ & $12(21 \%)$ & $1(1 \%)$ \\
\hline FLUS $(\mathrm{n}=19,10 \%)$ & $2(4 \%)$ & $17(13 \%)$ \\
\hline FN $(\mathrm{n}=11,6 \%)$ & $3(5 \%)$ & $8(6 \%)$ \\
\hline SM $(\mathrm{n}=27,14 \%)$ & $9(16 \%)$ & $18(13 \%)$ \\
\hline $\mathbf{M}(\mathrm{n}=118,62 \%)$ & $29(52 \%)$ & $89(66 \%)$ \\
\hline ND $(\mathrm{n}=2,1 \%)$ & $1(2 \%)$ & $1(1 \%)$ \\
\hline Total $(\mathrm{n}=190)$ & 56 & 134 \\
\hline
\end{tabular}

NIFTP= Noninvasive follicular thyroid neoplasm with papillary-like nuclear features 
Table 3. Histologic Types of Non-incidental Micronodules $(>0.5 \mathrm{~cm}$ and $\leq 1 \mathrm{~cm})$

\begin{tabular}{|c|c|c|c|c|c|}
\hline FNA Diagnosis & PTC & FC & FVPTC & NIFTP & Other \\
\hline B $(\mathrm{n}=13)$ & 6 & 1 & 4 & 1 & 1 (MTC) \\
\hline FLUS $(\mathrm{n}=3)$ & 1 & & 1 & 1 & \\
\hline FN $(\mathrm{n}=3)$ & 1 & 1 & 1 & & \\
\hline SM $(\mathrm{n}=10)$ & 6 & & 3 & 1 & \\
\hline $\mathbf{M}(\mathrm{n}=29)$ & 23 & & 5 & & 1 (TCV) \\
\hline ND $(\mathrm{n}=2)$ & 1 & & & 1 & \\
\hline Total $(\mathrm{n}=60)$ & 38 & 2 & 14 & 4 & 2 \\
\hline
\end{tabular}

PTC=Papillary thyroid carcinoma; FC=Follicular carcinoma; FVPTC=Follicular variant of papillary thyroid carcinoma; MTC=Medullary thyroid carcinoma; TCV=Tall cell variant of papillary thyroid carcinoma 
Table 4. Histologic Types of Incidental Micronodules $(\leq 0.5 \mathrm{~cm})$

\begin{tabular}{|c|c|c|c|}
\hline FNA Diagnosis & PTC & FVPTC & Other (MTC) \\
\hline B $(\mathrm{n}=11)$ & 6 & 4 & 1 \\
\hline FLUS $(\mathrm{n}=15)$ & 7 & 8 & \\
\hline FN $(\mathrm{n}=11)$ & 7 & 4 & 1 \\
\hline SM $(\mathrm{n}=7)$ & 4 & 2 & 1 \\
\hline M $(\mathrm{n}=8)$ & 6 & 1 & 3 \\
\hline ND $(\mathrm{n}=3)$ & 2 & 1 & \\
\hline Total $(\mathrm{n}=55)$ & 32 & 20 & \\
\hline
\end{tabular}

